\title{
A Survey of Hochschild Cohomology for von Neumann Algebras
}

\author{
Allan M. Sinclair and Roger R. Smith \\ Abstract. In this paper we survey the field of Hochschild coho- \\ mology for von Neumann algebras. We describe the basic defini- \\ tions and results without assuming background knowledge beyond \\ some familiarity with von Neumann algebra theory. We offer no \\ formal proofs of results, although we give detailed references to the \\ literature. Instead, we concentrate on explaining the main tech- \\ niques and how they are applied to obtain the significant theorems \\ of the subject.
}

\section{Introduction}

The study of cohomology in the algebraic context was initiated by Hochschild in a series of papers $[\mathbf{1 8}, \mathbf{1 9}, \mathbf{2 0}]$. This was subsequently adapted to the Banach algebra and operator algebra settings in $[\mathbf{2 1}, \mathbf{2 3}$, 25, 26] where continuity of multilinear maps was taken into account in the functional analytic framework. In this paper we survey the current state of knowledge in this field with particular reference to von Neumann algebras. There are detailed accounts of the basic results in an early survey by Ringrose, [41], and a later set of lecture notes

1991 Mathematics Subject Classification. Primary 46L10; Secondary 46L07.

Key words and phrases. von Neumann algebra, $C^{*}$-algebra, cohomology group, complete boundedness, Cartan, property $\Gamma$.

This paper is an expanded version of a talk given by the second author in the special session "Operator Algebras, Quantization, and Noncommutative Geometry: A Centennial Celebration in Honor of J. V. Neumann and M. H. Stone" at the Baltimore A.M.S. meeting, January 2003. 
by the authors, $[\mathbf{4 3}]$. For this reason we have omitted proofs from this work and have instead tried to give general ideas as to why results are true. While we have included all the main theorems for completeness, we have given more weight to later developments where, in some cases, the papers are yet to appear. The reader wishing for an account with all details of proofs is referred to $[43]$. These lecture notes also contain a discussion of the various applications of cohomology to which we felt that we could not do full justice here (see also $[\mathbf{2 2}, \mathbf{4 0}]$ ). We now give a brief desciption of the contents of the various sections; all definitions will be given at the appropriate places subsequently.

The starting point for continuous (or bounded) cohomology is a theorem, due independently to Kadison and Sakai, $[\mathbf{2 4}, 42]$, which states that every derivation $\delta: \mathcal{M} \rightarrow \mathcal{M}$ on a von Neumann algebra $\mathcal{M}$ has the form $\delta(x)=x m-m x$ for some fixed $m \in \mathcal{M}$, such derivations being called inner. The first cohomology group $H^{1}(\mathcal{M}, \mathcal{M})$ is the vector space of derivations modulo the inner derivations, so one interpretation of this theorem is that $H^{1}(\mathcal{M}, \mathcal{M})=0$ for all von Neumann algebras. It was then natural to conjecture the vanishing of the higher order groups $H^{n}(\mathcal{M}, \mathcal{M}), n \geq 2$. When $\mathcal{M}$ is faithfully represented on a Hilbert space $H, B(H)$ is then an $\mathcal{M}$-bimodule and the cohomology groups $H^{n}(\mathcal{M}, B(H))$ are also defined. In all known cases these groups are 0 , but in general the situation of $\mathcal{M}$ as the bimodule is better understood. It really suffices to study these problems for factors with separable predual. Direct integral theory allows us to pass to separably acting von Nuemann algebras, and there is a general procedure in [43] for extending to arbitrary von Neumann algebras. In Section 2 we present the definitions of the cohomology groups and we also discuss complete boundedness of linear and multilinear maps on $C^{*}$-algebras. In the last fifteen years this topic has become the main technical tool for resolving cohomological questions about von Neumann algebras. Cohomology and complete boundedness have enjoyed a symbiotic relationship where advances in one have triggered progress in the other.

In Section 3 we give the basic results of the early theory from $[23,25,26]$. Since the cohomology groups are quotients of spaces of cocycles by spaces of coboundaries, a given cocycle may be modified 
by adding a suitable coboundary to reach an equivalent cocycle with desirable properties. This is usually achieved by averaging a given cocycle over a specially chosen amenable group of unitary operators in the von Neumann algebra $\mathcal{M}$. This may need to be done several times but the net result, and probably the most important theorem in the subject, is that each cocycle is equivalent to a cocycle which is separately normal in each variable and which vanishes when any of its arguments lies in a specified hyperfinite von Neumann subalgebra $\mathcal{R}$. In particular, this implies that such a cocycle is $\mathcal{R}$-multimodular (see (2.8)-(2.10)). This is Theorem 3.3, which has the consequence that whenever $\mathcal{M}$ is hyperfinite and $\mathcal{V}$ is an arbitrary dual normal $\mathcal{M}$-bimodule then the cohomology groups $H^{n}(\mathcal{M}, \mathcal{V})$ are 0 for all $n \geq 1$, [23]. As shown by Connes, $[\mathbf{1 3}]$, this property characterizes hyperfiniteness.

The results of Section 3 summarize the state of knowledge up to the mid 1980's. At that point the emerging field of completely bounded maps was applied to cohomology theory, $[\mathbf{5}, \mathbf{9}, \mathbf{1 0}, \mathbf{1 1}]$, and we discuss this in Section 4. The coboundary operator preserves complete boundedness, and so a parallel theory of completely bounded cohomology can be developed. The decisive difference is that completely bounded maps have a very useful structure theory, discovered first in the linear case, $[\mathbf{1 5}, \mathbf{3 0}, \mathbf{3 3}]$, and subsequently for multilinear operators, $[\mathbf{9}, \mathbf{3 1}]$. This allowed the determination of $H_{c b}^{n}(\mathcal{M}, B(H)),[\mathbf{5}]$, and $H_{c b}^{n}(\mathcal{M}, \mathcal{M}),[\mathbf{1 1}, \mathbf{1 2}]$, for $n \geq 1$, and both sets of groups were found to vanish. These results, Theorems 4.1 and 4.3 , give a clear path to the original problem of bounded cohomology: try to show that a given cocycle is equivalent to one which is completely bounded. This works perfectly for von Neumann algebras of types $\mathrm{I}, \mathrm{II}_{\infty}$ or III; type I can be handled by the previously discussed hyperfiniteness results while types $\mathrm{II}_{\infty}$ and III are stable under tensoring with $B(H)$ which is enough to obtain complete boundedness of cocycles. Those factors of type $\mathrm{II}_{1}$ which are stable under tensoring with the hyperfinite type $\mathrm{II}_{1}$ factor $\mathcal{R}$ (the McDuff factors of $[\mathbf{2 7}, \mathbf{2 8}]$ ) are amenable to the same analysis. Not all type $\mathrm{II}_{1}$ factors have this property, and this case is not yet fully understood. Sections 5 and 6 contain recent results on 
further classes of type $\mathrm{II}_{1}$ algebras, and the common theme in both is complete boundedness.

The fifth section discusses the case of a separably acting type $\mathrm{II}_{1}$ von Neumann algebra with a Cartan subalgebra. This is a maximal abelian self-adjoint subalgebra $\mathcal{A}$ whose normalizer $\mathcal{N}(\mathcal{A})$ generates $\mathcal{M}$. An important consequence is that $\mathcal{A}$ and its reflection $J \mathcal{A} J$ on the Hilbert space $H=L^{2}(\mathcal{M}, \operatorname{tr})$, where $J$ is the conjugate linear isometry corresponding to the adjoint, together generate a masa in $B(H),[\mathbf{1 6}, \mathbf{3 7}]$. In combination with the Grothendieck inequality (5.1), this was sufficient to prove complete boundedness of $n$-cocycles into $\mathcal{M}$ for $n=2,3,[\mathbf{3 4}, \mathbf{6}]$. Refinements of these techniques lead to the general result of Theorem 5.4, [44], where suitable cocycles are shown to be completely bounded in the last variable which is sufficient to conclude that $H^{n}(\mathcal{M}, \mathcal{M})=0$ for $n \geq 4$. In the last section we move to the case of factors with property $\Gamma,[\mathbf{7}, \mathbf{8}]$. Once again vanishing of cohomology, Theorem 6.6, is obtained through the use of complete boundedness. Some earlier partial results are to be found in $[\mathbf{3}, \mathbf{1 1}]$, but the full resolution of this case rests on a strengthened form of Grothendieck's inequality, Theorem 6.2 , as well as on a $\|\cdot\|_{2}-$ norm joint continuity result, Theorem 6.4, which may be of independent interest.

We conclude with a remark on the results presented in this paper. In all cases we have shown that the cohomology groups under consideration vanush. While cohomology groups might be used as isomorphism invariants, they also act as obstructions to the von Neumann algebras having certain desirable properties. Vanishing of the cohomology groups should be viewed in the positive light of making these obstructions disappear.

\section{Preliminaries}

Let $\mathcal{A}$ be a $C^{*}$-algebra and let $\mathcal{V}$ be a Banach space which is also a two-sided $\mathcal{A}$-bimodule. To avoid pathologies, we will assume that

$$
\|x v\|,\|v x\| \leq\|v\|\|x\|, \quad x \in \mathcal{A}, v \in \mathcal{V}
$$

and that $1 v=v 1=v$ whenever $\mathcal{A}$ has an identity element. This is true for all of the main examples below. For any $n \geq 1, \mathcal{A}^{n}$ will denote 
the $n$-fold Cartesian product of copies of $\mathcal{A}$, and the space of bounded $n$-linear maps $\phi: \mathcal{A}^{n} \rightarrow \mathcal{V}$ will be denoted by $\mathcal{L}^{n}(\mathcal{A}, \mathcal{V})$. For $n=0$, we let $\mathcal{L}^{0}(\mathcal{A}, \mathcal{V})$ be $\mathcal{V}$. The coboundary map $\partial: \mathcal{L}^{n}(\mathcal{A}, \mathcal{V}) \rightarrow \mathcal{L}^{n+1}(\mathcal{A}, \mathcal{V})$ is defined as follows. For $n=0, \partial v$ is the derivation $x \mapsto x v-v x$, $x \in \mathcal{A}$. When $n \geq 1$ and $\phi \in \mathcal{L}^{n}(\mathcal{A}, \mathcal{V}), \partial \phi \in \mathcal{L}^{n+1}(\mathcal{A}, \mathcal{V})$ is defined by

$$
\begin{aligned}
\partial \phi\left(x_{1}, \ldots, x_{n+1}\right)= & x_{1} \phi\left(x_{2}, \ldots, x_{n}\right) \\
& +\sum_{i=1}^{n-1}(-1)^{i} \phi\left(x_{1}, \ldots, x_{i-1}, x_{i} x_{i+1}, x_{i+2}, \ldots, x_{n}\right) \\
& +(-1)^{n} \phi\left(x_{1}, \ldots, x_{n}\right) x_{n+1},
\end{aligned}
$$

for $x_{i} \in \mathcal{A}, 1 \leq i \leq n+1$. An algebraic computation establishes that $\partial \partial=0$. Thus the image of $\partial: \mathcal{L}^{n-1}(\mathcal{A}, \mathcal{V}) \rightarrow \mathcal{L}^{n}(\mathcal{A}, \mathcal{V})$ is contained in the kernel of $\partial: \mathcal{L}^{n}(\mathcal{A}, \mathcal{V}) \rightarrow \mathcal{L}^{n+1}(\mathcal{A}, \mathcal{V})$, and the quotient of these two vector spaces is the $n^{\text {th }}$ Hochschild cohomology group $H^{n}(\mathcal{A}, \mathcal{V})$. The elements of the kernel and image above are called respectively cocycles and coboundaries. For $n=1$, the cocycles are derivations, since the defining equation (2.2) can be rearranged to

$$
\phi\left(x_{1} x_{2}\right)=x_{1} \phi\left(x_{2}\right)+\phi\left(x_{1}\right) x_{2}, \quad x_{1}, x_{2} \in \mathcal{A}
$$

The coboundaries are the inner derivations implemented by elements of $\mathcal{V}$, and $H^{1}(\mathcal{A}, \mathcal{V})$ becomes the space of bounded derivations modulo the space of inner derivations.

One may impose further restrictions on the elements of $\mathcal{L}^{n}(\mathcal{A}, \mathcal{V})$ beyond boundedness. Provided that the extra conditions are compatible with the coboundary map, various cohomology theories arise by using the definition given above. We will discuss here the additional requirement of complete boundedness which leads to the completely bounded cohomology groups $H_{c b}^{n}(\mathcal{A}, \mathcal{V})$ for suitable modules $\mathcal{V}$.

The matrix algebras $\mathbb{M}_{n}(\mathcal{A})$ over a $C^{*}$-algebra $\mathcal{A}$ have canonical $C^{*}$-algebra norms obtained by faithfully representing $\mathcal{A}$ on a Hilbert space $H$ and identifying each $\mathbb{M}_{n}(\mathcal{A})$ with a $C^{*}$-algebra of operators on the $n$-fold sum $H^{n}$. A bounded map $\phi: \mathcal{A} \rightarrow \mathcal{B}$ between $C^{*}$-algebras induces a sequence of bounded maps $\phi_{n}: \mathbb{M}_{n}(\mathcal{A}) \rightarrow \mathbb{M}_{n}(\mathcal{B}), n \geq 1$, by tensoring $\phi$ with the identity on $\mathbb{M}_{n}(\mathbb{C})$, and $\phi$ is completely bounded 


$$
\|\phi\|_{\mathrm{cb}}:=\sup \left\{\left\|\phi_{n}\right\|: n \geq 1\right\}<\infty .
$$

This was generalized to multilinear maps in $[\mathbf{9}]$ by using matrix multiplication to replace the identity map on $\mathbb{M}_{n}(\mathbb{C})$. We illustrate this in the bilinear case which then has an obvious general extension. If $\phi: \mathcal{A} \times$ $\mathcal{B} \rightarrow B(H)$ is a bounded bilinear map then $\phi_{n}: \mathbb{M}_{n}(\mathcal{A}) \times \mathbb{M}_{n}(\mathcal{B}) \rightarrow$ $\mathbb{M}_{n}(B(H))$ is defined for matrices $\left(a_{i j}\right) \in \mathbb{M}_{n}(\mathcal{A}),\left(b_{i j}\right) \in \mathbb{M}_{n}(\mathcal{B})$ by specifying the $(i, j)$ entry of $\phi_{n}\left(\left(a_{i j}\right),\left(b_{i j}\right)\right)$ to be $\sum_{k=1}^{n} \phi\left(a_{i k}, b_{k j}\right)$. Then $\phi$ is said to be completely bounded if the analogous supremum to (2.4) is finite. The importance of completely bounded maps is two-fold: most naturally occurring maps on $C^{*}$-algebras are completely bounded and there is a very useful representation theory for such maps which is lacking for bounded maps. In the linear case a bounded map $\phi: \mathcal{A} \rightarrow B(H)$ is completely bounded precisely when it may be expressed as

$$
\phi(x)=V \pi(x) W, \quad x \in \mathcal{A},
$$

where $\pi: \mathcal{A} \rightarrow B(K)$ is a $*$-representation and $V: K \rightarrow H, W: H \rightarrow$ $K$ are bounded operators satisfying $\|V\|=\|W\|=\|\phi\|_{\mathrm{cb}}^{1 / 2},[30]$. The multilinear version, proved in $[\mathbf{9}]$ and extended to operator spaces in [31], is as follows. For clarity, we state it in the bilinear case, but the general case requires only more *-representations and connecting operators.

TheOREM 2.1. Let $\mathcal{A}_{1}, \mathcal{A}_{2}$ be $C^{*}$-algebras. A bounded bilinear map $\phi: \mathcal{A}_{1} \times \mathcal{A}_{2} \rightarrow B(H)$ is completely bounded if and only if it may be expressed by

$$
\phi(x, y)=V_{1} \pi_{1}(x) V_{2} \pi_{2}(y) V_{3}, \quad x \in \mathcal{A}_{1}, y \in \mathcal{A}_{2},
$$

where $\pi_{i}: \mathcal{A}_{i} \rightarrow B\left(K_{i}\right), i=1,2$, are $*$-representations and $V_{3}: H \rightarrow$ $K_{2}, V_{2}: K_{2} \rightarrow K_{1}, V_{1}: K_{1} \rightarrow H$ are bounded operators satisfying

$$
\left\|V_{1}\right\|=\left\|V_{2}\right\|=\left\|V_{3}\right\|=\|\phi\|_{\mathrm{cb}}^{1 / 3} \text {. }
$$

This theorem makes it an easy exercise to verify that the coboundary operator preserves complete boundedness, and so the completely bounded cohomology groups $H_{c b}^{n}(\mathcal{A}, \mathcal{V})$ can be defined whenever $\mathcal{V}$ has 
an operator space structure with respect to which it is also an operator $\mathcal{A}$-module. In particular, when $\mathcal{M} \subseteq B(H)$ is a von Neumann algebra, we will discuss $H_{c b}^{n}(\mathcal{M}, \mathcal{M})$ and $H_{c b}^{n}(\mathcal{M}, B(H))$ subsequently.

If $\mathcal{A} \subseteq \mathcal{B} \subseteq B(H)$ is an inclusion of $C^{*}$-algebras and $\phi: \mathcal{B} \times \mathcal{B} \rightarrow$ $B(H)$ is a bounded bilinear map then we say that $\phi$ is $\mathcal{A}$-modular if the following relations are satisfied:

$$
\begin{aligned}
& a \phi\left(b_{1}, b_{2}\right)=\phi\left(a b_{1}, b_{2}\right), \\
& \phi\left(b_{1} a, b_{2}\right)=\phi\left(b_{1}, a b_{2}\right), \\
& \phi\left(b_{1}, b_{2} a\right)=\phi\left(b_{1}, b_{2}\right) a,
\end{aligned}
$$

for $a \in \mathcal{A}$ and $b_{1}, b_{2} \in \mathcal{B}$. We omit the obvious extension to multilinear maps.

In anticipation of subsequent developments, we mention that we will be able to work with cocycles which are $\mathcal{A}$-modular with respect to a suitable subalgebra $\mathcal{A}$. Usually this means one with an amenable generating subgroup of unitaries or, for von Neumann algebras, a hyperfinite subfactor. When $\mathcal{M}$ is a factor of type $\mathrm{I}_{\infty}, \mathrm{II}_{\infty}$ or III, there is a factor $\mathcal{N}$ and a separable Hilbert space $H$ such that $\mathcal{M}$ is isomorphic to $\mathcal{N} \bar{\otimes} B(H)$. Any multilinear map $\phi$ on $\mathcal{M}$ which is separately normal in each variable and also modular with respect to the subalgebra $1 \otimes B(H)$ can be expressed as $\psi \otimes I$ where $\psi$ is multilinear on $\mathcal{N}$. Since $B(H)$ contains arbitrarily large matrix subalgebras, it is easy to establish the complete boundedness of $\psi$ and hence of $\phi$ in this situation. A similar argument applies to those type $\mathrm{II}_{1}$ factors which are stable under tensoring with the hyperfinite type $\mathrm{II}_{1}$ factor, but it should be noted that not all type $\mathrm{II}_{1}$ factors have this property.

\section{Reduction of cocycles}

In this section we will discuss a basic technique, originating in [23], which seeks to modify a given cocycle by a coboundary so that the resulting cocycle has some desirable properties. To motivate the approach, we first consider a von Neumann algebra $\mathcal{M}$ with a module $\mathcal{V}$ and we let $\mathcal{A}$ be a finite dimensional subalgebra of $\mathcal{M}$. Then $\mathcal{A}$ has a norm compact unitary group $\mathcal{U}$, whose normalized Haar measure 
we denote by $\mu$. Let $\phi: \mathcal{M} \times \mathcal{M} \rightarrow \mathcal{V}$ be a 2-cocycle, and define $\psi \in \mathcal{L}^{1}(\mathcal{M}, \mathcal{V})$ by

$$
\psi(x)=\int_{\mathcal{U}} u \phi\left(u^{*}, x\right) d \mu(u), \quad x \in \mathcal{M},
$$

where this integral converges in the norm topology of $\mathcal{V}$. Then

$$
\partial \psi(v, x)=\int_{U} v u \phi\left(u^{*}, x\right)-u \phi\left(u^{*}, v x\right)+u \phi\left(u^{*}, v\right) x d \mu(u),
$$

for $v \in \mathcal{U}$ and $x \in \mathcal{M}$, and invariance of the Haar measure allows us to replace $u$ by $v^{*} u$ in the first term. This equation then becomes

$$
\partial \psi(v, x)=\int_{\mathcal{U}} u\left[\phi\left(u^{*} v, x\right)-\phi\left(u^{*}, v x\right)+\phi\left(u^{*}, v\right) x\right] d \mu(u) .
$$

Since $\partial \phi\left(u^{*}, v, x\right)=0$, we may use the identity

$$
u^{*} \phi(v, x)-\phi\left(u^{*} v, x\right)+\phi\left(u^{*}, v x\right)-\phi\left(u^{*}, v\right) x=0
$$

to rewrite (3.3) as

$$
\partial \psi(v, x)=\int_{\mathcal{U}} u\left[u^{*} \phi(v, x)\right] d \mu(u)=\phi(v, x),
$$

for $v \in \mathcal{U}, x \in \mathcal{M}$. Then $\theta=\phi-\partial \psi$ is an equivalent cocycle with the additional property that $\theta(a, x)=0$ for all $a \in \mathcal{A}$ and $x \in \mathcal{M}$, since $\mathcal{A}$ is the linear span of $\mathcal{U}$. For $a \in \mathcal{A}, x, y \in \mathcal{M}$, the identity

$$
a \theta(x, y)-\theta(a x, y)+\theta(a, x y)-\theta(a, x) y=0
$$

shows that

$$
a \theta(x, y)=\theta(a x, y)
$$

since the last two terms in (3.6) vanish. Now define $\xi \in \mathcal{L}^{1}(\mathcal{M}, \mathcal{V})$ by

$$
\xi(x)=\int_{\mathcal{U}} \theta\left(x u, u^{*}\right) d \mu(u), \quad x \in \mathcal{M} .
$$

Then

$$
\partial \xi(x, y)=\int_{\mathcal{U}} x \theta\left(y u, u^{*}\right)-\theta\left(x y u, u^{*}\right)+\theta\left(x u, u^{*}\right) y d \mu(u) .
$$

If we replace $x$ by $a \in \mathcal{A}$ in (3.9) then

$$
\partial \xi(a, y)=\int_{U} a \theta\left(y u, u^{*}\right)-\theta\left(a y u, u^{*}\right) d \mu(u)=0,
$$


using (3.7) to obtain vanishing of the integrand. Now replace $y$ by $v \in \mathcal{U}$ in (3.9). This gives

$$
\partial \xi(x, v)=\int_{\mathcal{U}} x \theta\left(v u, u^{*}\right)-\theta\left(x v u, u^{*}\right)+\theta\left(x u, u^{*}\right) v d \mu(u) .
$$

The first term on the right vanishes and the second may be replaced by $\theta\left(x u, u^{*} v\right)$ using invariance of the Haar measure. The cocycle identity

$$
x u \theta\left(u^{*}, v\right)-\theta(x, v)+\theta\left(x u, u^{*} v\right)-\theta\left(x u, u^{*}\right) v=0,
$$

in which the first term is 0 , allows us to rewrite (3.11) as

$$
\partial \xi(x, v)=\int_{U}-\theta(x, v) d \mu(u)=-\theta(x, v) .
$$

If we define $\zeta$ to be $\theta+\partial \xi$, then these calculations show that $\zeta$ is a cocycle equivalent to $\phi$ but with the additional property that $\zeta(x, y)=$ 0 whenever at least one of the arguments is in $\mathcal{A}$. As a consequence, $\phi$ may be replaced by an equivalent cocycle $\zeta$ which is $\mathcal{A}$-modular. To obtain this extra information, consider the identity

$$
x \zeta(a, y)-\zeta(x a, y)+\zeta(x, a y)-\zeta(x, a) y=0
$$

for $x, y \in \mathcal{M}$ and $a \in \mathcal{A}$. The first and last terms vanish leaving

$$
\zeta(x a, y)=\zeta(x, a y) .
$$

The equation $\zeta(x, y a)=\zeta(x, y) a$ follows similarly from $\partial \zeta(x, y, a)=0$.

For simplicity, we have only discussed the 2-cocycle case, but these techniques are generally valid and give the following.

TheOREM 3.1. Let $\phi$ be an $n$-cocycle on $\mathcal{M}$ and let $\mathcal{A}$ be a finite dimensional $C^{*}$-subalgebra of $\mathcal{M}$. Then there exists $\psi \in \mathcal{L}^{n-1}(\mathcal{M}, \mathcal{V})$ such that $\phi-\partial \psi$ vanishes whenever any of its arguments lie in $\mathcal{A}$, and $\phi-\partial \psi$ is $\mathcal{A}$-modular.

In order to extend this result to more general subalgebras, certain restrictions must be placed on the modules. Henceforth we will assume that the module $\mathcal{V}$ is a dual normal module in the sense that $\mathcal{V}$ is a dual Banach space and the left and right actions, viewed as maps of $\mathcal{M} \times \mathcal{V}$ and $\mathcal{V} \times \mathcal{M}$ into $\mathcal{V}$ are separately continuous for the ultraweak topology on $\mathcal{M}$ and the $w^{*}$-topology on $\mathcal{V}$. This is the case when $\mathcal{V}$ 
is $\mathcal{M}$ or $B(H)$. This allows us to average over amenable rather than just compact unitary subgroups of $\mathcal{M}$. If $\mu$ is an invariant mean on the bounded continuous functions on an amenable group $\mathcal{U}$ of unitaries in $\mathcal{M}$, then $\mu$ applied to the function

$$
u \mapsto \omega\left(u \phi\left(u^{*}, x\right)\right), \quad \omega \in \mathcal{V}_{*},
$$

defines an element of $\left(\mathcal{V}_{*}\right)^{*}=\mathcal{V}$ for each $x \in \mathcal{M}$, and thus a bounded linear map $\psi \in \mathcal{L}^{1}(\mathcal{M}, \mathcal{V})$. Averaging over $\mathcal{U}$ in this way gives a result analogous to Theorem 3.1.

Theorem 3.2. Let $\mathcal{V}$ be a dual normal $\mathcal{M}$-module and let $\mathcal{A} \subseteq \mathcal{M}$ be a $C^{*}$-subalgebra which is the norm closed span of an amenable group $\mathcal{U}$ of unitaries. Then any $n$-cocycle $\phi$ is equivalent to an $n$-cocycle $\xi$ with the property that $\xi$ vanishes whenever one of the arguments lies in $\mathcal{A}$. Moreover $\xi$ is $\mathcal{A}$-modular.

The two main situations where this result is applied are as follows. If $\mathcal{A}$ is abelian, then its full unitary group is amenable. If $\mathcal{M}$ contains a hyperfinite subfactor $\mathcal{R}$, then $\mathcal{R}$ is the ultraweak closure of an increasing sequence of matrix subalgebras $\mathcal{A}_{n}$ with compact unitary groups $\mathcal{U}_{n}$, and their union $\mathcal{U}$ is amenable. This does not give immediately $\mathcal{R}$-modularity, but rather modularity with respect to the norm closed span of $\mathcal{U}$ which is ultraweakly dense in $\mathcal{R}$. Further subtle arguments are required to achieve $\mathcal{R}$-modularity. The full details may be found in $[43]$ and we will content ourselves with a statement of results.

The coboundary operator respects separate ultraweak continuity of multilinear maps (also referred to as separate normality) and so there are cohomology groups $H_{w}^{n}(M, V)$ where the relevant maps are required to be separately normal. By applying the averaging techniques above and using a result on the continuous extension of ultraweakly continuous cocycles, we arrive at the basic theorem of cohomology theory.

Theorem 3.3. (a) Let $\mathcal{M}$ be a von Neumann algebra with a dual normal module $\mathcal{V}$, and let $\mathcal{R}$ be a hyperfinite subalgebra of $\mathcal{M}$. Then each $n$-cocycle $\phi$ on $\mathcal{M}$ is equivalent to one which is separately normal and $\mathcal{R}$-modular. Moreover, if $\phi$ is separately normal and $\phi=\partial \psi$ for 
some $\psi \in \mathcal{L}^{n-1}(\mathcal{M}, \mathcal{V})$, then $\psi$ may be taken to be separately normal. As a consequence

$$
H^{n}(\mathcal{M}, \mathcal{V})=H_{w}^{n}(\mathcal{M}, \mathcal{V}), \quad n \geq 1
$$

(b) If $\mathcal{A}$ is an ultraweakly dense $C^{*}$-subalgebra of $\mathcal{M}$ then

$$
H^{n}(\mathcal{A}, \mathcal{V})=H_{w}^{n}(\mathcal{A}, \mathcal{V})=H^{n}(\mathcal{M}, \mathcal{V})=H_{w}^{n}(\mathcal{M}, \mathcal{V}), \quad n \geq 1
$$

If $\mathcal{M}$ is hyperfinite then this theorem shows that any $n$-cocycle into a dual normal $M$-module $\mathcal{V}$ is equivalent to one which vanishes whenever one of the arguments is in $\mathcal{M}$. This says, of course, that any cocycle is a coboundary and $H^{n}(\mathcal{M}, \mathcal{V})=0$ in this case, [23]. In [13], a converse to this result was given.

THEOREM 3.4. If $\mathcal{M}$ is a von Neumann algebra and $H^{1}(\mathcal{M}, \mathcal{V})=0$ for all dual normal $\mathcal{M}$-modules $\mathcal{V}$, then $\mathcal{M}$ is hyperfinite.

\section{Completely bounded cohomology}

In this section we discuss the completely bounded cohomology groups $H_{c b}^{n}(\mathcal{M}, B(H))$ and $H_{c b}^{n}(\mathcal{M}, \mathcal{M})$, where $\mathcal{M}$ is a von Neumann algebra on a Hilbert space $H$. Both groups will be 0 . The techniques for showing this are different in the two cases, but both depend crucially on the representation theorem for completely bounded multilinear maps (Theorem 2.1). The methods from Section 3, which show that any cocycle is equivalent to one which is separately normal, preserve complete boundedness. Thus, when considering a fixed completely bounded cocycle, it may be assumed to be separately normal and, if necessary, to be $\mathcal{R}$-modular for a fixed hyperfinite subalgebra $\mathcal{R}$ of $\mathcal{M}$. In particular, a cocycle may always be assumed to vanish when one of its arguments is a scalar.

Consider now a von Neumann algebra $\mathcal{M} \subseteq B(H)$ and let $\phi: \mathcal{M} \times$ $\mathcal{M} \rightarrow B(H)$ be a 2-cocycle such that $\phi(1, y)=\phi(x, 1)=0$ for all $x, y \in \mathcal{M}$. If $\phi$ is also completely bounded then there is a representation

$$
\phi(x, y)=V_{1} \pi_{1}(x) V_{2} \pi_{2}(y) V_{3}, \quad x, y \in \mathcal{M},
$$

where $\pi_{i}: \mathcal{M} \rightarrow B\left(K_{i}\right), i=1,2$, are $*$-representations and $V_{3}: H \rightarrow$ $K_{2}, V_{2}: K_{2} \rightarrow K_{1}$ and $V_{1}: K_{1} \rightarrow H$ are bounded operators. Let $P$ be 
the projection in $B\left(K_{1}\right)$ onto the closed linear span of $\left\{V_{2} \pi_{2}(y) V_{3} h: y \in\right.$ $\mathcal{M}, h \in H\}$. Then

$$
\phi(x, y)=V_{1} \pi_{1}(x) P V_{2} \pi_{2}(y) V_{3},
$$

and the condition $\phi(1, y)=0$ gives

$$
V_{1} P V_{2} \pi_{2}(y) V_{3} h=0, \quad y \in \mathcal{M}, h \in H,
$$

and thus $V_{1} P=0$. Replacing $V_{2}$ by $P V_{2}$ allows us to assume that $V_{1} V_{2}=0$. If we let $Q$ be the projection in $B\left(K_{2}\right)$ onto the closed linear span of $\left\{V_{2}^{*} \pi_{1}\left(x^{*}\right) V_{1}^{*} h: x \in \mathcal{M}, h \in H\right\}$ then we may replace $V_{2}$ by $V_{2} Q$, and the condition $\phi(x, 1)=0$ implies that $Q V_{3}=0$, arguing as above. Thus we may also assume that $V_{2} V_{3}=0$. Suppressing the representations $\pi_{1}$ and $\pi_{2}$ for notational convenience, (4.2) becomes

$$
\begin{aligned}
\phi(x, y) & =V_{1} x V_{2} y V_{3} \\
& =\left(V_{1} x-x V_{1}\right)\left(V_{2} y V_{3}\right) \\
& =\left(V_{1} x-x V_{1}\right)\left(V_{2} y-y V_{2}\right) V_{3} \\
& =\left[V_{1}, x\right]\left[V_{2}, y\right] V_{3},
\end{aligned}
$$

where $[\cdot, \cdot]$ denotes the commutator. A simple algebraic calculation shows that

$$
0=\partial \phi(x, y, z)=-\left[V_{1}, x\right]\left[V_{2}, y\right]\left[V_{3}, z\right] .
$$

To show that $\phi$ is a coboundary, we first let $e$ be the smallest projection such that $e\left[V_{3}, z\right]=\left[V_{3}, z\right]$, and define

$$
\psi(x)=V_{1} x V_{2}(1-e) V_{3}, \quad x \in \mathcal{M} .
$$

Then

$$
\begin{aligned}
\partial \psi(x, y) & =x V_{1} y V_{2}(1-e) V_{3}-V_{1} x y V_{2}(1-e) V_{3}+V_{1} x V_{2}(1-e) V_{3} y \\
& =-\left[V_{1}, x\right] y V_{2}(1-e) V_{3}+\left[V_{1}, x\right] V_{2}(1-e) V_{3} y \\
& =-\left[V_{1}, x\right]\left(\left[y, V_{2}\right](1-e) V_{3}+V_{2} y(1-e) V_{3}-V_{2}(1-e) V_{3} y\right) \\
& =\phi(x, y)-\left[V_{1}, x\right] V_{2}(1-e)\left[y, V_{3}\right] \\
& =\phi(x, y) .
\end{aligned}
$$


In this calculation we have used the fact that $\left[V_{1}, x\right]\left[V_{2}, y\right] e=0$ and that the space spanned by $\left[V_{3}, y\right] H$ is $\pi_{2}(\mathcal{M})$-invariant, so that $e$ and $1-e$ lie in $\pi_{2}(\mathcal{M})^{\prime}$.

The discussion above shows that $H_{c b}^{2}(\mathcal{M}, B(H))=0$, and we have included the argument to illustrate the importance of the completely bounded representation theorem. Similar calculations give a general result, $[5]$.

Theorem 4.1. Let $\mathcal{M} \subseteq B(H)$ be a von Neumann algebra. Then $H_{c b}^{n}(\mathcal{M}, B(H))=0$ for $n \geq 1$.

When a cocycle $\phi$ as above maps into $\mathcal{M}$, Theorem 4.1 shows that $\phi=\partial \psi$ for some $\psi \in \mathcal{L}_{c b}^{1}(\mathcal{M}, B(H))$ but not necessarily lying in $\mathcal{L}_{c b}^{1}(\mathcal{M}, \mathcal{M})$. Thus one cannot conclude immediately that $H_{c b}^{n}(\mathcal{M}, \mathcal{M})=$ 0 . This is true, however, but requires a preparatory averaging theorem, $[11,12]$, which can also be found in $[43]$.

The objective is to construct a contractive projection $\rho$ from the space $C B(\mathcal{M}, \mathcal{M})$ of completely bounded maps of $\mathcal{M}$ to itself onto the subspace $C B(\mathcal{M}, \mathcal{M})_{\mathcal{M}}$ of right $\mathcal{M}$-module maps. For a special class of maps this is easy to achieve. If $s, t \in \mathcal{M}$ and $\phi(x)=s x t$ then the Dixmier approximation theorem can be applied to $t$. Maps of the form

$$
\frac{1}{n} \sum_{i=1}^{n} \phi\left(x u_{i}\right) u_{i}^{*}=\frac{1}{n} \sum_{i=1}^{n} s x u_{i} t u_{i}^{*}, \quad x \in \mathcal{M},
$$

where the $u_{i}$ 's are unitaries in $\mathcal{M}$, converge strongly to a map $x \mapsto s x z$ where $z$ is a central element of $\mathcal{M}$, and this is a right $\mathcal{M}$-module map. This motivates the correct approach which is to consider sets of operators $\left\{u_{\alpha}\right\}$ in $\mathcal{M}$ for which $\Sigma u_{\alpha} u_{\alpha}^{*}=1$ strongly, and to then examine the resulting completely bounded maps $x \mapsto \Sigma \phi\left(x u_{\alpha}\right) u_{\alpha}^{*}$. The desired projection is obtained from a minimal invariant set argument, and the representation theorem for completely bounded maps is crucial. We refer to [43] for the many details which we are omitting here, but once the method works on the right it is easy to obtain a projection onto left module maps by taking adjoints, and then onto bimodule maps by applying these two results sequentially. We summarize: 
Theorem 4.2. For a von Neumann algebra $\mathcal{M}$, there exist contractive projections of $C B(\mathcal{M}, \mathcal{M})$ onto the spaces $C B(\mathcal{M}, \mathcal{M})_{\mathcal{M}},{ }_{\mathcal{M}} C B(\mathcal{M}, \mathcal{M})$ and $C B_{\mathcal{M}}(\mathcal{M}, \mathcal{M})$ of respectively right, left, and two-sided $\mathcal{M}$-modular completely bounded maps.

We can immediately apply this result to cohomology. Let $\rho$ be the projection onto right module maps obtained above, and note that every such map has the simple form $x \mapsto t x$ for some $t \in \mathcal{M}$. The nature of the construction also shows that if $\phi \in C B(\mathcal{M}, \mathcal{M}), a \in \mathcal{M}$, and $\phi_{a}(x)=\phi(a x)$, then $\left(\rho \phi_{a}\right)(x)=(\rho \phi)(a x)$. If we have a completely bounded bilinear map $\theta(x, z)$ and we regard $z$ as the variable and fix $x$, then there is a completely bounded map $\psi(x)$ such that

$$
\rho \theta(x, z)=\psi(x) z, \quad x, z \in \mathcal{M},
$$

and

$$
\rho \theta(x y, z)=\psi(x y) z, \quad \rho \theta(x, y z)=\psi(x) y z .
$$

If $\theta$ is, additionally, a 2-cocycle then we may apply $\rho$ to the $z$ variable in the cocycle identity

$$
x \theta(y, z)-\theta(x y, z)+\theta(x, y z)-\theta(x, y) z=0
$$

to obtain

$$
x \psi(y) z-\psi(x y) z+\psi(x) y z-\theta(x, y) z=0,
$$

from which $\theta(x, y)=\partial \psi(x, y)$ follows by letting $z=1$. This argument applies equally to $n$-cocycles which are completely bounded and leads to the following result, $[\mathbf{1 1}]$.

THEOREM 4.3. If $\mathcal{M}$ is a von Neumann algebra, then $H_{c b}^{n}(\mathcal{M}, \mathcal{M})=$ 0 for $n \geq 1$.

It is important to note that, in order to apply $\rho$, only complete boundedness in the last variable is required. Similarly complete boundedness in the first variable would allow us to apply the projection onto left module maps with the same effect.

We observed in Section 2 that if $\mathcal{M}$ is stable under tensoring with $B(H)$ (or the hyperfinite $\mathrm{II}_{1}$ factor $\mathcal{R}$ ) then any separately normal multilinear map which is modular with respect to these distinguished 
subalgebras is completely bounded. Since cocycles are equivalent to ones which are separately normal and modular with respect to a chosen hyperfinite subfactor, Theorem 4.3 applies in these cases, $[\mathbf{5 , 1 1}]$.

TheOREM 4.4. If $\mathcal{M}$ is a von Neumann algebra of type $\mathrm{I}, \mathrm{II}_{\infty}$, III, or is type $\mathrm{II}_{1}$ and stable under tensoring with $\mathcal{R}$, then $H^{n}(\mathcal{M}, \mathcal{M})=0$ and $H^{n}(\mathcal{M}, B(H))=0$ for $n \geq 1$.

Not all type $\mathrm{II}_{1}$ factors are stable under tensoring with $\mathcal{R}$ since the fundamental group need not be $\mathbb{R}^{+},[\mathbf{3 8}]$, and so this theorem leaves

open the type $\mathrm{II}_{1}$ case. In the following sections we will discuss this further.

\section{Algebras with Cartan subalgebras}

In view of the results of the previous section, the main open problems in cohomology theory concern type $\mathrm{II}_{1}$ factors. The questions of whether $H^{n}(\mathcal{M}, \mathcal{M})=0, n \geq 2$, and $H^{n}(\mathcal{M}, B(H))=0, n \geq 1$, remain unresolved but there has been recent progress on both in special cases. In this section we consider the first of these questions for type $\mathrm{II}_{1}$ von Neumann algebras $\mathcal{M}$ with Cartan subalgebras. A maximal abelian subalgebra $\mathcal{A} \subseteq \mathcal{M}$ is said to be Cartan if $\mathcal{M}$ is generated as a von Neumann algebra by those unitaries $u \in \mathcal{M}$ which normalize $\mathcal{A}$ in the sense that $u \mathcal{A} u^{*}=\mathcal{A}$. Such unitaries constitute the normalizer $\mathcal{N}(\mathcal{A})$ of $\mathcal{A}$ in $\mathcal{M}$. Cartan subalgebras arise naturally in the theory of crossed products. When a discrete group $G$ acts freely and ergodically on an abelian von Neumann algebra $\mathcal{A}$ without atoms, the resulting crossed product $\mathcal{A} \times{ }_{\alpha} G$ is a type $\mathrm{II}_{1}$ factor and $\mathcal{A}$ is a Cartan subalgebra; the hypotheses on the action guarantee that $\mathcal{A}$ is a masa, and $\mathcal{N}(\mathcal{A})$ contains both the unitaries implementing the action of $G$ and the unitary group of $\mathcal{A}$, thus generating $\mathcal{A} \times{ }_{\alpha} G$.

We will require some deep results in order to discuss the Cartan subalgebra case. The first of these is the non-commutative Grothendieck inequality proved by Pisier, [32], and further refined by Haagerup, [17], whose version we give here. Let $\theta: \mathcal{M}^{2} \rightarrow \mathbb{C}$ be a separately normal bounded bilinear form on a von Neumann algebra $\mathcal{M}$. Then there exist 
normal states $f_{1}, f_{2}, g_{1}, g_{2}$ on $\mathcal{M}$ such that

$$
|\theta(x, y)| \leq \frac{1}{2}\|\theta\|\left(f_{1}\left(x^{*} x\right)+f_{2}\left(x x^{*}\right)+g_{1}\left(y^{*} y\right)+g_{2}\left(y y^{*}\right)\right) .
$$

If $\mathcal{R}$ is a hyperfinite subalgebra whose relative commutant $\mathcal{R}^{\prime} \cap \mathcal{M}$ equals the center $\mathcal{Z}$ of $\mathcal{M}$, then this inequality can be strengthened for bilinear maps which are inner $\mathcal{R}$-modular in the sense that

$$
\theta(x r, y)=\theta(x, r y), \quad r \in \mathcal{R}, \quad x, y \in \mathcal{M}
$$

Fix an amenable group of unitaries $\mathcal{U}$ which generates $\mathcal{R}$, and replace $x$ and $y$ in (5.1) by respectively $x u$ and $u^{*} y$ for $u \in \mathcal{U}$. Then average over $\mathcal{U}$, the result being

$$
|\theta(x, y)| \leq\|\theta\|\left(F\left(x x^{*}\right)+G\left(y^{*} y\right)\right), \quad x, y \in \mathcal{M}
$$

where $F$ and $G$ are normal states. When $u^{*} x^{*} x u$ is averaged over $\mathcal{U}$, it becomes $\mathbb{E}\left(x^{*} x\right)=\mathbb{E}\left(x x^{*}\right)$ where $\mathbb{E}$ is the conditional expectation onto $\mathcal{Z}$, explaining why the $x^{*} x$ term has disappeared from (5.3), as has the $y y^{*}$ term for the same reason. A consequence of (5.3) is the following result.

TheOREM 5.1. Let $\mathcal{M}$ be a type $\mathrm{II}_{1}$ von Neumann algebra with a hyperfinite subalgebra $\mathcal{R}$ with $\mathcal{R}^{\prime} \cap \mathcal{M}=\mathcal{Z}$. Let $\psi: \mathcal{M} \times \mathcal{M} \rightarrow \mathcal{M}$ be a separately normal inner $\mathcal{R}$-modular map. Then

$$
\left\|\sum_{i=1}^{n} \psi\left(x_{i}, y_{i}\right)\right\| \leq 2\|\psi\|\left\|\sum_{i=1}^{n} x_{i} x_{i}^{*}\right\|^{1 / 2}\left\|\sum_{i=1}^{n} y_{i}^{*} y_{i}\right\|^{1 / 2}
$$

for any finite sets of elements $x_{i}, y_{i} \in \mathcal{M}$.

There is a standard technique for applying bilinear results to linear maps. If $\phi: \mathcal{M} \rightarrow \mathcal{M}$ is a bounded linear map then define $\psi: \mathcal{M} \times$ $\mathcal{M} \rightarrow \mathcal{M}$ by

$$
\psi(x, y)=\phi(x) \phi\left(y^{*}\right)^{*}, \quad x, y \in \mathcal{M}
$$

If $\phi$ is right $\mathcal{R}$-modular then $\psi$ is inner $\mathcal{R}$-modular. By letting $y_{i}$ be $x_{i}^{*}$ in (5.4), we obtain the following. 
Theorem 5.2. Let $\mathcal{R} \subseteq \mathcal{M}$ satisfy the hypotheses of Theorem 5.1, and let $\phi: \mathcal{M} \rightarrow \mathcal{M}$ be a bounded normal right $\mathcal{R}$-modular map. Then, for $x_{1}, \ldots, x_{n} \in \mathcal{M}$,

$$
\left\|\sum_{i=1}^{n} \phi\left(x_{i}\right) \phi\left(x_{i}\right)^{*}\right\| \leq 2\|\phi\|^{2}\left\|\sum_{i=1}^{n} x_{i} x_{i}^{*}\right\| .
$$

We can give an alternative formulation of this theorem in terms of row bounded maps, which are intermediate between bounded and completely bounded maps. Parallel to the definition of a completely bounded map, $\phi: \mathcal{M} \rightarrow \mathcal{M}$ is row bounded if

$$
\|\phi\|_{\mathrm{r}}=\sup \left\{\left\|\left(\phi\left(x_{1}\right), \ldots, \phi\left(x_{n}\right)\right)\right\|:\left\|\left(x_{1}, \ldots, x_{n}\right)\right\| \leq 1\right\}<\infty
$$

where the rows in (5.7) are allowed to be arbitrarily long.

Corollary 5.3. If $\phi: \mathcal{M} \rightarrow \mathcal{M}$ is bounded, separately normal, and right $\mathcal{R}$-modular for a hyperfinite subalgebra $\mathcal{R}$ with $\mathcal{R}^{\prime} \cap \mathcal{M}=\mathcal{Z}$, then $\phi$ is row bounded and

$$
\|\phi\| \leq\|\phi\|_{\mathrm{r}} \leq \sqrt{2}\|\phi\| .
$$

This corollary allows us to obtain a useful estimate on the projection $\rho$ of Theorem 4.2. If $\phi: \mathcal{M} \rightarrow \mathcal{M}$ is normal, completely bounded and right $\mathcal{R}$-modular for $\mathcal{R}^{\prime} \cap \mathcal{M}=\mathcal{Z}$, then expressions of the form

$$
\Sigma \phi\left(x u_{\alpha}\right) u_{\alpha}^{*}: \Sigma u_{\alpha} u_{\alpha}^{*} \leq 1
$$

are bounded in norm by $\|\phi\|_{\mathrm{r}}\|x\|$. Right $\mathcal{R}$-modularity then gives the estimate

$$
\|\rho \phi\| \leq\|\phi\|_{\mathrm{r}} \leq \sqrt{2}\|\phi\|
$$

from (5.8). It is curious that complete boundedness of $\phi$ is apparently required to construct $\rho$, but $\|\phi\|_{\mathrm{cb}}$ is not required in (5.10).

We can now state the main result of this section which has evolved through the special cases of $[\mathbf{3 4}, \mathbf{6}]$ to its final formulation in $[\mathbf{4 4}]$. We will then sketch the main steps in proving it, referring to $[\mathbf{4 4}, \mathbf{4 5}]$ for the full details.

Theorem 5.4. Let $\mathcal{M}$ be a separably acting type $\mathrm{II}_{1}$ von Neumann algebra with a Cartan subalgebra $\mathcal{A}$. Then $H^{n}(\mathcal{M}, \mathcal{M})=0$ for $n \geq 1$. 
By work of Popa, $[\mathbf{3 6}]$, in the factor case and extended in $[\mathbf{4 6}]$ to the general case, there is a hyperfinite subalgebra $\mathcal{R}$ such that $\mathcal{A} \subseteq \mathcal{R} \subseteq \mathcal{M}$ and $\mathcal{R}^{\prime} \cap \mathcal{M}=\mathcal{Z}$ (separable predual is essential here). We may restrict our attention to a separately normal cocycle $\phi$ which is $\mathcal{R}$-modular, and the results of Section 3 show that we need only demonstrate that it is a coboundary on the $C^{*}$-algebra generated by the normalizer $\mathcal{N}(\mathcal{A})$. Again, for simplicity, we shall confine ourselves to the case of a 2cocycle.

As observed by Rădulescu, [39], elements of $\mathcal{A}$ can be pulled through a cocycle in certain circumstances. If $u \in \mathcal{N}(\mathcal{A})$, then $\mathcal{R}$-modularity (and thus $\mathcal{A}$-modularity) of $\phi$ leads to

$$
\begin{aligned}
a \phi(u, x) b & =\phi(a u, x b)=\phi\left(u u^{*} a u, x b\right) \\
& =\phi(u, \beta(a) x b), \quad a, b \in \mathcal{A}, \quad x \in \mathcal{M},
\end{aligned}
$$

where $\beta$ is the $*$-automorphism $a \mapsto u^{*} a u$ of $\mathcal{A}$. Cartan subalgebras have the property that they norm their containing von Neumann algebras in the sense that, for $X \in \mathbb{M}_{n}(\mathcal{M})$,

$$
\|X\|=\sup \|R X C\|,
$$

where the supremum is taken over rows and columns in $\mathbb{M}_{n}(\mathcal{A})$ of unit norm, [35]. Equation (5.11) lifts to the $n \times n$ matrix level where, modulo the norm preserving automorphism $\beta$, rows and columns over $\mathcal{A}$ can be pulled through to the second entry. Using this fact, it is then straightforward to show that $\phi(u, x)$ is completely bounded as a linear function of $x$ when $u \in \mathcal{N}(\mathcal{A})$, and thus also when the first argument is in the algebra generated by $\mathcal{N}(\mathcal{A})$. Since these maps are right $\mathcal{R}$-modular, by $\mathcal{R}$-modularity of $\phi$, the estimate of (5.10) allows us to apply $\rho$ to $\phi(m, x)$, when $m \in \operatorname{Alg}(\mathcal{N}(\mathcal{A}))$, in a continuous way, and thus when $m$ lies in the completion $C^{*}(\mathcal{N}(\mathcal{A}))$. This is sufficient to conclude that $\phi$ is a coboundary, due to the remarks immediately before and after Theorem 4.3. It is interesting to note that we do not know whether the hypotheses are sufficient to imply complete boundedness of the bilinear map, but this property in the last variable is all that is required. 


\section{Factors with property $\Gamma$}

In this section $\mathcal{M}$ will denote a separably acting type $\mathrm{II}_{1}$ factor with property $\Gamma$. This property was introduced by Murray and von Neumann [29], in order to distinguish the hyperfinite factor $\mathcal{R}$ from the factor arising from the free group on two generators. The defining condition is as follows: given $x_{1}, \ldots, x_{n} \in \mathcal{M}$ and $\varepsilon>0$, there exists a unitary $u \in \mathcal{M}, \operatorname{tr} u=0$, such that

$$
\left\|\left[u, x_{i}\right]\right\|_{2}<\varepsilon, \quad 1 \leq i \leq n,
$$

where $\operatorname{tr}$ is the unique normalized normal trace on $\mathcal{M}$ and $\|x\|_{2}=$ $\left(\operatorname{tr}\left(x^{*} x\right)\right)^{1 / 2}$. Later, Dixmier, [14], gave an equivalent formulation of property $\Gamma$ by the following condition: given an integer $n$, elements $x_{1}, \ldots, x_{m} \in \mathcal{M}$ and $\varepsilon>0$, there exist $n$ orthogonal projections $p_{1}, \ldots, p_{n} \in \mathcal{M}$, each of trace $n^{-1}$, such that their sum is 1 and

$$
\left\|\left[p_{i}, x_{j}\right]\right\|_{2}<\varepsilon, \quad 1 \leq i \leq n, \quad 1 \leq j \leq m .
$$

This second formulation will prove to be the more useful one here. By fixing a $\|\cdot\|_{2}$-dense countable set of elements in $\mathcal{M}$ and letting $\varepsilon$ be $k^{-1}$ for $k \geq 1$, we may find a countable set of projections in $\mathcal{M}$ so that (6.2) can always be satisfied by projections from this set. We will refer to such a collection as a set of Dixmier projections. An important step is to be able to find such a set for $\mathcal{M}$ within a hyperfinite subfactor. This can be achieved by using a result of Popa, $[\mathbf{3 6}]$, stating that any separably acting type $\mathrm{II}_{1}$ factor $\mathcal{M}$ contains a hyperfinite subfactor $\mathcal{R}$ of trivial relative commutant $\mathcal{R}^{\prime} \cap \mathcal{M}=\mathbb{C} 1$. In [7], this was employed to deduce a stronger version in the case of a property $\Gamma$ factor.

THEOREM 6.1. Let $\mathcal{M}$ be a separably acting type $\mathrm{II}_{1}$ factor with property $\Gamma$. Then $\mathcal{M}$ contains a hyperfinite subfactor $\mathcal{R}$ of trivial relative commutant and, moreover, $\mathcal{R}$ can be chosen to contain a set of Dixmier projections for $\mathcal{M}$.

Each of the two conditions is easy to achieve separately, but obtaining them simultaneously requires a lengthy argument for which we refer to [7]. The basic idea is to construct $\mathcal{R}$ from an inductively defined sequence $\mathcal{A}_{1} \subseteq \mathcal{B}_{1} \subseteq \mathcal{A}_{2} \subseteq B_{2} \ldots$ of matrix subfactors of $\mathcal{M}$. The $\mathcal{A}_{i}$ 's 
are used to build a full set of Dixmier projections into $\mathcal{R}$ (the weak closure of the union), while the $\mathcal{B}_{i}$ 's are included to ensure that $\mathcal{R}$ has trivial relative commutant. Henceforth we may and will assume that a hyperfinite subfactor $\mathcal{R}$ of $\mathcal{M}$ with property $\Gamma$ satisfies the conclusions of this theorem.

Before considering the cohomology problem for factors with property $\Gamma$, we discuss two auxiliary results which are valid for general type $\mathrm{II}_{1}$ factors. The first of these concerns a type of Grothendieck inequality for multilinear maps which is based on the standard bilinear version. We let $\operatorname{tr}_{n}$ denote the unique normalized trace on $\mathbb{M}_{n}(\mathcal{M})$ and we introduce a new norm by

$$
\rho_{n}(X)=\left(\|X\|^{2}+n \operatorname{tr}_{n}\left(X^{*} X\right)\right)^{1 / 2}, \quad X \in \mathbb{M}_{n}(\mathcal{M}) .
$$

We also fix a hyperfinite subfactor $\mathcal{R}$ of trivial relative commutant. There is no direct generalization of the Grothendieck inequality to three or more variables, and the essential difference appears to be that a bilinear map $\phi\left(x_{1}, x_{2}\right)$ has only outer variables while a trilinear map $\phi\left(x_{1}, x_{2}, x_{3}\right)$ has an inner variable $x_{2}$ which is hard to reach. However, useful inequalities can be obtained by fixing some variables and applying a vector functional to connect with (5.3). We illustrate this for a trilinear map $\phi: \mathcal{M}^{3} \rightarrow B(H)$ which is separately normal and $\mathcal{R}$-multimodular. To handle the outer variables we consider bilinear maps

$$
\psi_{1}(x, y)=\phi\left(x^{*}, x_{2}, x_{3}\right)^{*} \phi\left(y, x_{2}, x_{3}\right), \quad x, y \in \mathcal{M}
$$

and

$$
\psi_{3}(x, y)=\phi\left(x_{1}, x_{2}, x\right) \phi\left(x_{1}, x_{2}, y^{*}\right)^{*}, \quad x, y \in \mathcal{M},
$$

whereas the appropriate map for the inner variable is

$$
\psi_{2}(x, y)=\phi\left(x, y, x_{3}\right), \quad x, y \in \mathcal{M} .
$$

For arbitrary vectors $\xi, \eta \in H$, the bilinear forms

$$
\theta_{i}(x, y)=\left\langle\psi_{i}(x, y) \xi, \eta\right\rangle, \quad 1 \leq i \leq 3, \quad x, y \in \mathcal{M},
$$

are inner $\mathcal{R}$-modular and separately normal, so that the inequality (5.3) is valid. This approach applies equally to the amplifications $\phi_{n}$ 
to $\mathbb{M}_{n}(\mathcal{M})$, and repeated averaging over $\mathcal{R} \otimes \mathbb{M}_{n}$ leads to the following result.

THEOREM 6.2. Let $M$ be a type $\mathrm{II}_{1}$ factor with a hyperfinite subfactor of trivial relative commutant and let $\phi: \mathcal{M}^{k} \rightarrow B(H)$ be a separately normal $\mathcal{R}$-multimodular map. Then, for each $n \geq 1$ and $X_{1}, \ldots, X_{k} \in \mathbb{M}_{n}(\mathcal{M})$,

$$
\left\|\phi_{n}\left(X_{1}, \ldots, X_{k}\right)\right\| \leq 2^{k / 2}\|\phi\| \rho_{n}\left(X_{1}\right) \ldots \rho_{n}\left(X_{k}\right) .
$$

The term $n \operatorname{tr}_{n}\left(X^{*} X\right)$ in the definition of $\rho_{n}(X)$ precludes (6.8) from implying complete boundedness of $\phi$ in Theorem 6.2, although this is our objective when $\mathcal{M}$ has property $\Gamma$. A step in this direction is to observe that if $P \in \mathbb{M}_{n}(\mathcal{M})$ is a projection of trace $n^{-1}$, then

$$
\rho_{n}(X P)^{2}=\|X P\|^{2}+n \operatorname{tr}_{n}\left(P X^{*} X P\right) \leq 2\|X\|^{2} .
$$

This gives

COROllary 6.3. In addition to the hypotheses of Theorem 6.2, let $P \in \mathbb{M}_{n}(\mathcal{M})$ be a projection of trace $n^{-1}$. Then, for $X_{1}, \ldots, X_{k} \in$ $\mathbb{M}_{n}(\mathcal{M})$

$$
\left\|\phi_{n}\left(X_{1} P, \ldots, X_{k} P\right)\right\| \leq 2^{k}\|\phi\|\left\|X_{1}\right\| \ldots\left\|X_{k}\right\|
$$

To illustrate the relevance of this, and to motivate what comes next, consider a normal linear map $\phi: \mathcal{M} \rightarrow B(H)$ on a property $\Gamma$ factor which is modular with respect to a hyperfinite subfactor $\mathcal{R}$ containing a set of Dixmier projections for $\mathcal{M}$. Let $p \in \mathcal{R}$ be any projection. Then

$$
\begin{aligned}
p \phi(x) & =p \phi(p x)=p \phi([p, x])+p \phi(x p) \\
& =\phi(p[p, x])+p \phi(x p) p, \quad x \in \mathcal{M} .
\end{aligned}
$$

This algebraic identity is valid at the $n^{\text {th }}$ matrix level with $p$ replaced by $P=p \otimes I_{n}$. Moreover, we may find a countable set of projections in $\mathbb{M}_{n}(\mathcal{R})$ of this form such that

$$
\operatorname{tr}_{n}\left(P_{i j}\right)=n^{-1}, \quad \sum_{j=1}^{n} P_{i j}=1, \quad \lim _{i \rightarrow \infty}\left\|\left[P_{i j}, X\right]\right\|_{2}=0
$$


for each $X \in \mathbb{M}_{n}(\mathcal{M})$. For an arbitrary pair of unit vectors $\xi, \eta \in H^{n}$, (6.11) becomes

$$
\begin{aligned}
\left\langle\phi_{n}(X) \xi, \eta\right\rangle & =\sum_{j=1}^{n}\left\langle P_{i j} \phi_{n}(X) \xi, \eta\right\rangle \\
& =\sum_{j=1}^{n}\left\langle\phi_{n}\left(P_{i j}\left[P_{i j}, X\right]\right) \xi, \eta\right\rangle+\sum_{j=1}^{n}\left\langle P_{i j} \phi\left(X P_{i j}\right) P_{i j} \xi, \eta\right\rangle,
\end{aligned}
$$

for $X \in \mathbb{M}_{n}(\mathcal{M})$ and $i \geq 1$. The last sum in (6.13) can be estimated by

$$
\max _{i, j}\left\|\phi_{n}\left(X P_{i j}\right)\right\| \leq 2\|\phi\|\|X\|
$$

by Corollary 6.3. The arguments in the penultimate sum in (6.13) are $P_{i j}\left[P_{i j}, X\right]$ which are uniformly bounded and tend to 0 in $\|\cdot\|_{2^{-}}$ norm as $i \rightarrow \infty$. Thus this sum vanishes in the limit provided that $\lim _{i \rightarrow \infty}\left\langle\phi_{n}\left(P_{i j}\left[P_{i j}, X\right]\right) \xi, \eta\right\rangle=0$, which certainly would result from $\|\cdot\|_{2^{-}}$ norm continuity of $\phi_{n}$ on bounded balls. However, this follows from normality and the Grothendieck inequality (see $[43,5.4 .3]$ and $[7$, Theorem 4.4]). Letting $i \rightarrow \infty$ in (6.13) results in the estimate

$$
\left\|\phi_{n}(X)\right\| \leq 2\|\phi\|\|X\|, \quad X \in \mathbb{M}_{n}(\mathcal{M}), \quad n \geq 1
$$

which establishes complete boundedness of $\phi$ with $\|\phi\|_{\mathrm{cb}} \leq 2\|\phi\|$.

To a certain point, this argument extends smoothly to the multilinear case. There are more complicated but similar analogs of (6.11) and (6.13) but the penultimate sum in (6.13) has, in the bilinear case, terms like $\left\langle\phi_{n}\left(\left[P_{i j}, X\right], P_{i j}\left[P_{i j}, Y\right]\right) \xi, \eta\right\rangle$. Successfully taking the limit as $i \rightarrow \infty$ now requires joint $\|\cdot\|_{2}$-norm continuity, rather than the separate $\|\cdot\|_{2}$-norm continuity guaranteed by separate normality. Baire, [1] , applied his category theorem to a separately continuous map $f:[0,1]^{2} \rightarrow[0,1]$ to conclude that there is a point $\left(x_{0}, y_{0}\right)$ of joint continuity for each $y_{0} \in[0,1]$. This extends to an arbitrary separately continuous function $f: \mathcal{X} \times \mathcal{Y} \rightarrow \mathcal{Z}$ on complete metric spaces (see [2] for a more general result). This is the starting point for the proof of the next result.

THEOREM 6.4. Let $\phi: \mathcal{M}^{k} \rightarrow B(H)$ be a bounded separately normal k-linear map. 
(a) If $\phi$ maps into $\mathcal{M}$, then $\phi$ is jointly $\|\cdot\|_{2}$-norm continuous when restricted to bounded balls.

(b) If $\xi, \eta \in H$ then the map

$$
\psi\left(x_{1}, \ldots, x_{k}\right)=\left\langle\phi\left(x_{1}, \ldots, x_{k}\right) \xi, \eta\right\rangle, \quad x_{i} \in \mathcal{M},
$$

is jointly $\|\cdot\|_{2}$-norm continuous when restricted to bounded balls.

The result mentioned before the theorem is used to obtain joint continuity in the $\|\cdot\|_{2}$-norm for a bilinear map $\phi$ at a point $(a, 0)$ in the product of two unit balls. Then joint continuity is deduced at $(0,0)$ and subsequently for all points $(x, y)$ in the unit balls. The multilinear case is proved by induction, regarding the $n$-fold product $B^{n}$ of balls as $B \times B^{n-1}$ and starting again with a point of joint continuity at $(a, 0, \ldots, 0)$.

By combining Corollary 6.3, Theorem 6.4, and by establishing algebraic identities analogous to (6.11) and (6.13), the following result is achieved. Note that while we require $\mathcal{M}$ to have a separable predual, equivalent to admitting a representation on a separable Hilbert space, we do not make any assumptions on $H$.

THEOREM 6.5. Let $\mathcal{M} \subseteq B(H)$ be a type $\mathrm{II}_{1}$ factor with a separable predual and property $\Gamma$. Let $\mathcal{R}$ be a hyperfinite subfactor with trivial relative commutant and containing a set of Dixmier projections for $\mathcal{M}$. Then any separately normal $\mathcal{R}$-modular $k$-linear map $\phi: \mathcal{M}^{k} \rightarrow B(H)$, $k \geq 1$, is completely bounded and satisfies the estimate $\|\phi\|_{\mathrm{cb}} \leq 2^{k}\|\phi\|$.

Since cocycles can always be assumed to be $\mathcal{R}$-modular and separately normal, this result gives the equalities

$$
H^{n}(\mathcal{M}, \mathcal{M})=H_{c b}^{n}(\mathcal{M}, \mathcal{M}), \quad H^{n}(\mathcal{M}, B(H))=H_{c b}^{n}(\mathcal{M}, B(H)),
$$

for any $n \geq 1$, at least when $\mathcal{M}$ has a separable predual (and, of course, property $\Gamma$ ). From Section 4, we conclude that these cohomology groups are 0 . To remove the requirement of a separable predual, a technical result is needed. This states that any property $\Gamma$ factor is the ultraweak closure of an increasing net of such factors with separable preduals. We may now summarize the discussion of this section. 
THEOREM 6.6. Let $\mathcal{M} \subseteq B(H)$ be a type $\mathrm{II}_{1}$ factor with property Г. Then, for $n \geq 1$,

$$
H^{n}(\mathcal{M}, \mathcal{M})=0 \quad \text { and } \quad H^{n}(\mathcal{M}, B(H))=0 .
$$

This theorem was proved in $[\mathbf{7}, \mathbf{8}]$ but is only new for $n \geq 3$. For $n=1$, the two cases are in $[\mathbf{2 4}, \mathbf{4 2}, \mathbf{3}]$ while for $n=2$ we refer to $[\mathbf{1 1}, \mathbf{4}]$. The approach taken in $[\mathbf{7}]$ has its origins in [4].

Sections 5 and 6 describe the most recent advances in this field but do not cover all type $\mathrm{II}_{1}$ factors. The factor $V N\left(\mathbb{F}_{2}\right)$ arising from the free group on two generators has neither property $\Gamma,[29]$, nor a Cartan subalgebra, [48], and the groups $H^{n}\left(V N\left(\mathbb{F}_{2}\right), V N\left(\mathbb{F}_{2}\right)\right), n \geq 2$, are yet to be determined. It would be very interesting to resolve this problem for at least the case $n=2$.

\section{References}

[1] R. Baire, Sur les fonctions de variables réelles, Ann. Mat. Pura Appl. 3 (1899), $1-122$.

[2] J.C. Breckenridge and T. Nishiura, Partial continuity, quasicontinuity, and Baire spaces, Bull. Inst. Math. Acad. Sinica 4 (1976), 191-203.

[3] E. Christensen, Similarities of $\mathrm{II}_{1}$ factors with property $\Gamma$, J. Operator Theory 15 (1986), 281-288.

[4] E. Christensen, Finite von Neumann algebra factors with property gamma, J. Funct. Anal. 186 (2001), 366-380.

[5] E. Christensen, E.G. Effros and A.M. Sinclair, Completely bounded multilinear maps and $C^{*}$-algebraic cohomology, Invent. Math. 90 (1987), 279-296.

[6] E. Christensen, F. Pop, A.M. Sinclair and R.R. Smith, On the cohomology groups of certain finite von Neumann algebras, Math. Ann. 307 (1997), 71-92.

[7] E. Christensen, F. Pop, A.M. Sinclair and R.R. Smith, Hochschild cohomology of factors with property $\Gamma$, Ann. of Math. (2) 158 (2003), 635-659.

[8] E. Christensen, F. Pop, A.M. Sinclair and R.R. Smith, Property $\Gamma$ factors and the Hochschild cohomology problem. Proc. Natl. Acad. Sci. USA 100 (2003), 3865-3869.

[9] E. Christensen and A.M. Sinclair, Representations of completely bounded multilinear operators, J. Funct. Anal. 72 (1987), 151-181.

[10] E. Christensen and A.M. Sinclair, A survey of completely bounded operators, Bull. London Math. Soc. 21 (1989), 417-448.

[11] E. Christensen and A.M. Sinclair, On the Hochschild cohomology for von Neumann algebras, unpublished manuscript. 
[12] E. Christensen and A.M. Sinclair, Module mappings into von Neumann algebras and injectivity, Proc. London Math. Soc. (3) 71 (1995), 618-640.

[13] A. Connes, On the cohomology of operator algebras, J. Funct. Anal. 28 (1978), 248-253.

[14] J. Dixmier, Quelques propriétés des suites centrales dans les facteurs de type $\mathrm{II}_{1}$, Invent. Math. 7 (1969), 215-225.

[15] E.G. Effros and Z.-J. Ruan, Operator spaces, London Mathematical Society Monographs, New Series, vol. 23, Oxford University Press, New York, 2000.

[16] J. Feldman and C. Moore, Ergodic equivalence relations, cohomology, and von Neumann algebras, I, II, Trans. Amer. Math. Soc. 234 (1977), 289-359.

[17] U. Haagerup, The Grothendieck inequality for bilinear forms on $C^{*}$-algebras, Adv. in Math. 56 (1985), 93-116.

[18] G. Hochschild, On the cohomology groups of an associative algebra, Ann. of Math. 46 (1945), 58-67.

[19] G. Hochschild, On the cohomology theory for associative algebras, Ann. of Math. 47 (1946), 568-579.

[20] G. Hochschild, Cohomology and representations of associative algebras, Duke Math. J. 14 (1947), 921-948.

[21] B.E. Johnson, Cohomology in Banach algebras, Mem. Amer. Math. Soc. 127, American Mathematical Society, Providence, R.I., 1972. iii+96 pp.

[22] B.E. Johnson, Perturbations of Banach algebras, Proc. London Math. Soc. (3) 34 (1977), 439-458.

[23] B.E. Johnson, R.V. Kadison and J.R. Ringrose, Cohomology of operator algebras. III. Reduction to normal cohomology, Bull. Soc. Math. France 100 (1972), 73-96.

[24] R.V. Kadison, Derivations of operator algebras, Ann. of Math. 83 (1966), 280-293.

[25] R.V. Kadison and J.R. Ringrose, Cohomology of operator algebras I. Type I von Neumann algebras, Acta Math. 126 (1971), 227-243.

[26] R.V. Kadison and J.R. Ringrose, Cohomology of operator algebras II. Extended cobounding and the hyperfinite case, Ark. Mat. 9 (1971), 55-63.

[27] D. McDuff, Central sequences and the hyperfinite factor, Proc. London Math. Soc. (3) 21 (1970), 443-461.

[28] D. McDuff, On residual sequences in a $\mathrm{II}_{1}$ factor, J. London Math. Soc. (2) 3 (1971), 273-280.

[29] F.J. Murray and J. von Neumann, On rings of operators IV, Ann. of Math. (2) 44 (1943), 716-808.

[30] V.I. Paulsen, Completely bounded maps and dilations, Pitman Research Notes in Mathematics Series, vol. 146, Longman Scientific \& Technical, Harlow, 1986. 
[31] V.I. Paulsen and R.R. Smith, Multilinear maps and tensor norms on operator systems, J. Funct. Anal. 73 (1987), 258-276.

[32] G. Pisier, Grothendieck's theorem for noncommutative $C^{*}$-algebras, with an appendix on Grothendieck's constants, J. Funct. Anal. 29 (1978), 397-415.

[33] G. Pisier, Introduction to operator space theory, London Mathematical Society Lecture Note Series, vol. 294, Cambridge University Press, Cambridge, 2003.

[34] F. Pop and R.R. Smith, Cohomology for certain finite factors, Bull. London Math. Soc. 26 (1994), 303-308.

[35] F. Pop, A.M. Sinclair and R.R. Smith, Norming $C^{*}$-algebras by $C^{*}$ subalgebras. J. Funct. Anal. 175 (2000), 168-196.

[36] S. Popa, On a problem of R.V. Kadison on maximal abelian $*$-subalgebras in factors, Invent. Math. 65 (1981), 269-281.

[37] S. Popa, Notes on Cartan subalgebras in type $\mathrm{II}_{1}$ factors, Math. Scand. 57 (1985), 171-188.

[38] S. Popa, On a class of type $\mathrm{II}_{1}$ factors with Betti numbers invariants, preprint, 2001.

[39] F. Rădulescu, Vanishing of $H_{w}^{2}(M, K(H))$ for certain finite von Neumann algebras, Trans. Amer. Math. Soc. 326 (1991), 569-584.

[40] I. Raeburn and J. Taylor, Hochschild cohomology and perturbations of Banach algebras, J. Funct. Anal. 25 (1977), 258-266.

[41] J. Ringrose, Linear mappings between operator algebras, Symposia Mathematica, Vol. XX (Convegno sulle Algebre $i C^{*}$ e loro Applicazioni in Fisica Teorica, Convegno sulla Teoria degli Operatori Indice e Teoria K, INDAM, Roma, 1975), pp. 297-315. Academic Press, London, 1976.

[42] S. Sakai, Derivations of $W^{*}$-algebras, Ann. of Math. (2) 83 (1966), 273-279.

[43] A.M. Sinclair and R.R. Smith, Hochschild cohomology of von Neumann algebras, London Mathematical Society Lecture Note Series, vol. 203, Cambridge University Press, Cambridge, 1995.

[44] A.M. Sinclair and R.R. Smith, Hochschild cohomology for von Neumann algebras with Cartan subalgebras, Amer. J. Math. 120 (1998), 1043-1057.

[45] A.M. Sinclair and R.R. Smith, The Hochschild cohomology problem for von Neumann algebras, Proc. Natl. Acad. Sci. USA 95 (1998), 3376-3379.

[46] A.M. Sinclair and R.R. Smith, Cartan subalgebras of finite von Neumann algebras, Math. Scand. 85 (1999), 105-120.

[47] A.M. Sinclair and R.R. Smith, Cohomology for finite index inclusions of factors, J. Operator Theory 44 (2000), 335-345.

[48] D. Voiculescu, The analogues of entropy and of Fisher's information measure in free probability theory III. The absence of Cartan subalgebras, Geom. Funct. Anal., 6 (1996), 172-199. 
Department of Mathematics, University of Edinburgh, Edinburgh, EH9 3JZ, SCOTLAND

E-mail address: A.Sinclair@ed.ac.uk

Department of Mathematics, Texas A\&M University, College StaTION, TX 77843, U.S.A.

E-mail address: rsmith@math.tamu.edu 